Cahiers d'études japonaises

\title{
Les principes de monumentalité dans l'architecture moderne : Analyse du discours architectural dans les premières œuvres de Tange Kenzō (1936-1962)
}

\section{Benoît Jacquet}

\section{OpenEdition}

Journals

Édition électronique

URL : https://journals.openedition.org/cipango/447

DOI : $10.4000 /$ cipango.447

ISSN : 2260-7706

Éditeur

INALCO

Édition imprimée

Date de publication : 1 janvier 2008

Pagination : 286-291

ISBN : 978-2-85837-177-4

ISSN : $1164-5857$

Référence électronique

Benoît Jacquet, «Les principes de monumentalité dans l'architecture moderne : Analyse du discours architectural dans les premières œuvres de Tange Kenzō (1936-1962) », Cipango [En ligne], 15 | 2008, mis en ligne le 14 novembre 2010, consulté le 30 juin 2021. URL : http://journals.openedition.org/ cipango/447 ; DOI : https://doi.org/10.4000/cipango.447

Ce document a été généré automatiquement le 30 juin 2021.

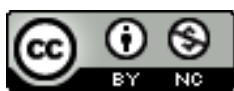

Cipango est mis à disposition selon les termes de la Licence Creative Commons Attribution - Pas d'Utilisation Commerciale 4.0 International. 


\title{
Les principes de monumentalité dans l'architecture moderne : Analyse du discours architectural dans les premières œuvres de Tange Kenzō (1936-1962)
}

\author{
Benoît Jacquet
}

\section{RÉFÉRENCE}

Les principes de monumentalité dans l'architecture moderne: Analyse du discours architectural dans les premières cuvres de Tange Kenzō (1936-1962), Thèse de doctorat soutenue par Benoît Jacquet (Paris-8, 2007), sous la direction de J.-L. Cohen et N. Fiévé.

1 La thèse de doctorat porte sur les principes de la monumentalité énoncés par les architectes du mouvement moderne au $\mathrm{Xx}^{\mathrm{e}}$ siècle. L'étude est centrée sur les premières œuvres de l'architecte japonais Tange Kenzō 丹下健三 (1913-2005) entre le milieu des années 1930 et le début des années 1960. Ces trois décennies essentielles pour la formation de l'architecture japonaise contemporaine englobent un événement des plus traumatisants de l'histoire du siècle passé : la Seconde Guerre mondiale.

2 En un volume de 674 pages de texte - incluant 30 pages de bibliographie (en langues occidentales et japonaise), 44 pages d'annexes (traductions de textes et d'entretiens), 135 illustrations et un index onomastique de 534 entrées - le point de vue de l'auteur s'appuie sur une analyse du discours architectural développé dans des textes théoriques (textes de réflexion, textes produits à l'occasion de concours, textes de circonstances, entretiens, articles de journaux) et dans la conception de projets pratiques d'architecture et d'urbanisme. 
3 La partie introductive de cette thèse de doctorat présente la méthode et la position scientifique adoptées par l'auteur - architecte diplômé de l'Ecole d'architecture de Paris-La Villette, de l'Ecole des hautes études en sciences sociales et de l'Université de Kyōto (master et doctorat) -, les hypothèses et les enjeux de sa recherche doctorale. Les mots clés de la thèse, le monument et ses dérivés sémantiques, le monumental et la monumentalité, sont ensuite définis en relation avec la notion à laquelle ils se trouvent historiquement associés : la modernité. L'auteur propose d'analyser les origines et les aboutissements de la monumentalité dans l'optique de la création architecturale contemporaine : depuis l'invention de la notion de monumentalité à la Renaissance par Alberti, à la question de la restauration du patrimoine (à partir du xix ${ }^{\mathrm{e}}$ siècle) et à l'arbitraire des formes du néo-classicisme qui tendent au «pseudo-monumental». La question actuelle étant celle des valeurs esthétiques et éthiques des monuments créés à l'époque contemporaine.

4 L'argument principal de la thèse, la question de la monumentalité dans le discours de l'architecture moderne, est ensuite présenté sous une perspective élargie à ses divers développements en Occident puis au Japon.

Dans la première partie de la thèse, le thème de la monumentalité est abordé suivant un contexte historique enveloppant les grands projets monumentaux du début du $\mathrm{xx}^{\mathrm{e}}$ siècle. La monumentalité y est présentée comme une caractéristique majeure des grands aménagements urbains, ceux qui ont servi les régimes totalitaires de l'entredeux-guerres (notamment en Allemagne et en Italie) autant que ceux qui ont représenté des valeurs démocratiques (comme le Plan de Washington). La monumentalité moderne va réinterpréter le vocabulaire de l'architecture classique, mais les architectes ont également eu recours à des formes et des discours novateurs. Les expressions de la monumentalité vont également être réinventées par l'évolution de l'architecture moderne, fondée sur les principes de rationalité et de fonctionnalité développés par l'industrialisation, orientée par les progrès sociaux et le développement de l'urbanisme, dotée d'un certain minimalisme ornemental en phase avec l'évolution de l'art et de l'esthétique de son temps. La formation de la scène architecturale suit un parcours similaire de l'Europe au Japon, au début du xxe siècle. Le cas nippon n'est ni le résultat d'une production exogène, ni le fruit d'une simple importation de valeurs et de pensées occidentales. A la suite des mouvements intellectuels, artistiques et littéraires du Japon du début de l'ère Shōwa, les mouvements d'architecture vont adapter, voire modifier, la modernité au projet d'identité nationale japonais.

6 L'architecture japonaise, qui va être étudiée puis réinterprétée par les architectes occidentaux, à commencer par Frank Lloyd Wright, a offert une alternative et de nouvelles approches formelle, matérielle et environnementale à l'architecture moderne. Cette ouverture à l'Orient, grâce au japonisme européen et aux orientalistes de Boston, était un retour de l'ouverture à la civilisation occidentale du Japon de Meiji. En regard, parfois en réaction, les architectes japonais, qui avaient évolué au gré des écoles et des styles occidentaux entre la fin du XIx ${ }^{e}$ siècle et le début de la colonisation de l'Asie orientale, vont peu à peu à revoir leurs propres traditions nationales. A partir des années 1930, ce retour à la tradition se conjugue avec une dérive idéologique nationaliste comparable à celle que vit alors l'Europe. La crise économique entraîna une crise des valeurs culturelles, un repli vers la figure totalitaire de l'Empereur et un rejet de l'Occident. La présentation des grandes tendances de l'architecture japonaise - des écoles dites expressionniste (hyōgen ha 表現派), Bauhaus (バウハウス派) et Le 
Corbusier (ル・コルビュジエ派) -, avant et pendant la guerre, permet de mieux comprendre les contextes historique, social, culturel et politique dans lesquels les architectes ont évolué.

7 L'œuvre de Tange Kenzō est représentative de la génération d'architectes qui va participer à toutes les étapes de transformations politique, sociale et physique du Japon contemporain : l'évolution de l'expansion colonialiste des années 1930 vers un conflit militaire mondial, les discours belliqueux devenus pacifiques après la guerre, la reconstruction puis la période dite de haute croissance économique. Entre les concours de monuments de guerre et ceux pour les Jeux Olympiques de Tōkyō, les principes de la monumentalité théorisée et mise en pratique dans l'architecture de Tange Kenzō se sont construits grâce à une réinterprétation des rapports fondamentaux à la tradition et à la création, à l'environnement et aux échelles du projet architectural et urbain. Ces principes sont devenus, au cours du siècle passé, des enjeux cruciaux de la création architecturale et de l'aménagement territorial.

8 La deuxième partie de la thèse est axée sur les principes d'une monumentalité «latente» dans les discours sur la tradition et la création de l'architecture moderne. Mâtiné des philosophies de Nietzsche et de Heidegger, se cherchant chez Michel-Ange et Le Corbusier d'incontestables mentors, Tange Kenzō passe ses années estudiantines sous l'égide des tenants d'une interprétation créative du classicisme. Entre les théories d'un Itō Chūta - premier architecte japonais "orientaliste » et révélateur du culte moderne au Hōryūji de Nara, symbole d'un héritage indo-européen de l'architecture bouddhique - et celles de Le Corbusier, Tange Kenzō se situe dans la lignée des architectes qui ont œuvré à l'invention d'une tradition d'architecture japonaise moderne.

9 En opposition aux doctrines et aux enseignements de l'Ecole des Beaux-Arts, les essais théoriques de Le Corbusier sur le Parthénon présentaient une approche plus abstraite des valeurs esthétiques de la monumentalité classique occidentale. Tange Kenzō qui forme sa pensée architecturale à la fin des années 1930, a cherché dans ce discours une origine référentielle pour la création d'une architecture japonaise contemporaine " classique ». Ses études sur la villa Katsura (xvil ${ }^{\mathrm{e}}$ siècle) et sur le sanctuaire d'Ise (vII siècle) s'inscrivent dans la lignée des discours modernistes qui ont participé à une légitimation de ces monuments en tant que modèles théoriques pour la création d'une monumentalité moderne, comme le Parthénon l'avait été pour les Occidentaux. A la suite d'Itō Chūta et des orientalistes de Boston qui, tels Ralph Adams Cram, virent des accents hellénistes dans les courbes des temples bouddhiques de Nara, les architectes japonais vont étudier le patrimoine selon un «regard moderne». Les livres de photographie de Kishida Hideto vont insister sur cette vision nouvelle du passé et redécouvrir les qualités esthétiques et fonctionnelles de l'architecture ancienne. En 1933, Bruno Taut sera guidé devant la villa impériale de Katsura, comme le sera ensuite Walter Gropius vingt ans plus tard, alors que cette architecture "simple et raffinée » est devenue une des figures privilégiées des débats controversés sur la tradition (dentō ronsō 伀統論争).

10 La démarche créative absolument moderne de Tange Kenzō porte également les traits d'une démarche " anti-moderne ", telle que la définit Antoine Compagnon. Alors que le Japon des années 1930 produit un effort de modernisation forcée, représentée par l'architecture «internationale » des architectes de l'école dite Bauhaus, son goût pour l'architecture japonaise, les formes baroques, Michel-Ange plutôt que De Vinci, la 
modénature et les matières nobles d'autrefois, fait de Tange un architecte dont on ne sait alors s'il serait à l'avant-garde ou à l'arrière-garde de son temps. Sa réflexion s'inscrit dans celle d'une époque de l'histoire du Japon influencée par des idéologies métissées d'occidentalisme ou d'orientalisme à rebours, par des débats sur l'« esprit japonais » et sur le «dépassement de la modernité 》(kindai no chōkoku 近代の超克) occidentale. Pendant la Guerre du Pacifique, les concours de mémoriaux, lui offriront une tribune idéale pour débuter sa carrière.

11 La troisième partie de la thèse analyse les principes de la monumentalité liés au concept d'environnement mis en pratique par Tange Kenzō à l'occasion des concours d'architecture organisés par l'Académie d'architecture du Japon (Nihon kenchiku gakkai 日本建築学会). Pendant l'été 1942, suivant les consignes du Bureau de planification (Kikakuin 企画院), le Comité architectural pour la Construction de la «Sphère de coprospérité de la Grande Asie orientale» (Daitōa kyōeiken 大東亜共栄圏) organisa un concours pour l'aménagement d'un mémorial dédié à sa cause (la Daitōa). Après leur échec au concours de Mémorial aux âmes loyales (chūreitō 忠霊塔) de 1939, les architectes modernistes ont décidé de revenir à un vocabulaire formel plus traditionaliste leur permettant de prendre la commande du parti architectural de la Daitōa.

12 La grande majorité des architectes japonais participèrent à ce concours. Tange Kenzō fut lauréat grâce à un projet de sanctuaire au pied du Mont Fuji. Ce sanctuaire, dont l'architecture se voulait une relecture de la spatialité antique japonaise, s'inspire des formes du sanctuaire shintō (jinja 神社) et du tertre funéraire impérial (kofun 古填). Inscrit dans un plan d'aménagement plus vaste, le sanctuaire est relié par un axe routier et ferroviaire à la Maison impériale (kōkyo 皇居), une sorte de chemin de pèlerinage (sandō 参道) le long de la route du Tōkaidō. Projet politique avant tout, son créateur démontrait sa capacité à coupler les tendances contradictoires de la tradition architecturale japonaise et d'un aménagement territorial contemporain.

La démarche conceptuelle de Tange Kenzō pour le Mémorial de la Daitōa est influencée par sa connaissance des projets urbains du Kikakuin, mais également par les recherches et débats sur la culture architecturale japonaise menés à cette époque. Maekawa Kunio 前川國男 (1905-1985), membre influent du jury de concours, inspiré par la problématique du philosophe Nishida Kitarō sur la culture japonaise (Nihon bunka no mondai, 1940), considérait à cette époque que la culture architecturale japonaise devait naître d'une tradition environnementale créative. Le projet de Mémorial de la Daitōa de son jeune disciple Tange se fonde sur une relation immanente et ontologique par rapport au culte de l'Empereur divin japonais. Il unifie matériellement (par un axe routier) et symboliquement le Palais impérial au Mont Fuji, la culture à la nature, dans un aménagement qui prend en compte les différentes échelles architecturales, urbaines et paysagères.

La dernière et quatrième partie de la thèse prolonge cette recherche d'un ordre environnemental par une étude des principes de monumentalité relatifs au concept d'échelle dans le Mémorial de la Paix à Hiroshima (1949-1955). Cette première œuvre construite par le laboratoire de Tange Kenzō à l'Université de Tōkyō est analysée en détail en suivant le long cours de sa genèse, depuis les premières heures du concours jusqu'à sa réalisation. Les sources documentaires étudiées proviennent des articles et des dessins d'architecture publiés sur ce projet ainsi que d'entretiens réalisés auprès de Tange Kenzō et de ses collaborateurs (par des historiens japonais ainsi que par l'auteur 
lui-même). La réalisation du Mémorial de la Paix (musée et parc) permit à Tange Kenzō de mettre en pratique son discours théorique dans un contexte politique complètement différent. Ce projet symbole d'une reconstruction pacifique et démocratique du Japon associe les valeurs esthétiques de la tradition japonaise dans un lieu qui unifie les échelles humaine, sociale et divine de l'architecture dans un grand aménagement urbain.

Comment l'architecte du projet de Mémorial de la Daitōa (non réalisé) a-t-il pu être également celui du Mémorial de la Paix à Hiroshima? La question reste en suspens et l'auteur n'a pas cherché à la réviser. Le travail mené par l'équipe de Tange Kenzō à Hiroshima (et dans d'autres villes japonaises) lui aura probablement permis de faire table rase d'un passé troublé.

L'implication de Tange Kenzō dans la reconstruction de Hiroshima a commencé en tant que consultant pour le Bureau de la Reconstruction des dommages dus à la guerre (sensai fukkōin 戦炎復興院). En 1948, Tange Kenzō avait également été lauréat du concours pour la Cathédrale catholique de Hiroshima (1948), avec un projet qui lui aussi était un exemple de la capacité de synthèse, même de syncrétisme, des formes de l'architectures sacrée - l'arche des basiliques romane, le sandō du sanctuaire shintō, le cloître (keidai 境内) du monastère bouddhique - dans une œuvre d'architecture d'expression moderne.

En conclusion, cette thèse entend démontrer que les premières œuvres de Tange Kenzō témoignent de la participation d'un architecte à l'histoire de l'architecture de la Seconde Guerre mondiale. Cette œuvre d'architecture, politique dans le sens étymologique du terme, nous permet de comprendre la formation intellectuelle d'un architecte qui est devenue une des figures tutélaires de l'architecture contemporaine. Premier architecte japonais «international », Tange Kenzō a été le chef de file d'une école représentée par les grands noms de l'architecture japonaise de la seconde moitié du vingtième siècle : des architectes comme Maki Fumihiko, Isozaki Arata, Kurokawa Kishō (1934-2007) qui ont perpétué la tradition d'une architecture japonaise qui serait essentiellement moderne.

L'étude s'arrête sur le projet de Plan pour Tōkyō proposé par le Laboratoire Tange Kenzō au début des années 1960, un grand plan d'urbanisme fondé sur des principes de croissance, d'axialité des voies de circulation et d'extension linéaire sur la mer et vers la montagne. Les grands principes de la monumentalité de ce projet sont issus de la recherche menée par Tange Kenzō pendant une trentaine d'années sur la monumentalité de l'architecture japonaise.

19 La cohérence de cette pensée architecturale démontre que cette monumentalité répondait aux enjeux de son temps, pour des raisons idéologiques, des questions d'identité nationale et de dépassement des modèles occidentaux, mais aussi pour des raisons politiques de contrôle de l'espace urbain. Ces principes - qui sont, premièrement, le dialogue entre la tradition et la création ; deuxièmement, le rapport à l'environnement; et, troisièmement, la corrélation entre les différentes échelles, architecturales, urbaines et paysagères - dépassent les clivages d'un pays et d'une époque. L'auteur a essayé de montrer la spécificité du cas japonais, mais aussi la correspondance de ces principes dans le monde occidental contemporain. 